\title{
Dengue: A Recent Challenge In Pakistan
}

\author{
Sannia Perwaiz Iqbal, Sajid Abbas Jaffri
}

Dengue is one of the fast emerging diseases of humans and is among the most widespread vector-borne tropical viral disease in the world today. The current incidence of dengue infections around the world is more than 58.4 million cases per year with mortality between 10,000 to 20,000. Countries in South Asia and South East Asia have the highest burden of this disease ${ }^{1}$. It is present in more than 128 countries around the world and nearly 4 billion people at the risk of developing this disease ${ }^{2}$.

The World Health Organization (WHO) has placed dengue among the 10 most global health problems. Climate change leading to increased flooding and rains, unusually hot and humid whether conditions in certain areas, inadequate control of mosquitos, increased urbanization and rapid growth in population, especially in developing countries are some of the major factors that have been contributing to spread of this disease ${ }^{3}$.

In Pakistan, 2019 has been the worst year in the country's history in which more than 44,000 cases have been reported by early November $2019^{4}$. However, many healthcare professionals believe that these numbers could be much higher as majority of cases in rural areas and slums are never reported.

Dengue fever/Dengue Hemorrhagic Fever is caused by an arbovirus belonging to the flaviviradae family. It is transmitted by the female mosquito Aedes, primarily aegypti and albopictus (daytime feeder) when it bites the human for her blood meal. The virus is a single stranded RNA and has 5 different types. All of these can cause severe illness which can be sometimes life threatening. Each serotype provides a specific lifetime immunity and a short-term cross- immunity against other serotypes. When the mosquito bites the human, the virus gets transferred into the blood stream, where it replicates, especially in target organs and lymphatic tissues. It is then again released and circulates in the patient's bloodstream. When a second mosquito bites the patient it ingests the virus. The virus replicates in the mosquito's midgut and infects its salivary glands. The mosquito carrying the virus then spreads the disease by biting other people. Incubation period is usually around 4 to 7 days (range, 3 to

Sannia Perwaiz Iqbal
Senior Instructor, Department of Medicine,
Bahria University Medical and Dental College
Email: sanniapi@gmail.com
Sajid Abbas Jaffri
Senior Professor, Head of Medicine Department,
Received: 04-12-2019

14 days). Clinical picture can vary from asymptomatic infection to severe life threatening illness. Most important risk factors for the development of severe disease include young age, female gender, prior infection, and certain genotypes of the virus 5 .

According to WHO, dengue is classified into 4 clinical syndromes, which include undifferentiated fever, classic dengue fever and Dengue Hemorrhagic Fever/Dengue Shock Syndrome.

The signs and symptoms of classic dengue disease include high grade fever, headache, pain behind eyes balls, severe pain in muscle and joints, nausea and vomiting, rashes on some body parts and dehydration.

Severe dengue occurs in less than $5 \%$ of the infected patients. It starts with non-specific symptoms but 4 to 7 days after the onset, plasma leakage with hemorrhagic features could develop causing hypoproteinemia, ascites, peripheral derma, pleural and cardiac effusions. There may be an increase in hematocrit by $20 \%$ and thrombocytopenia is also a characteristic feature. There can be bleeding from gums, nose, skin and gastrointestinal track. All of these can progress to severe circulatory collapse, manifested by feeble pulse, low blood pressure, and a pulse pressure of $<20 \mathrm{~mm} \mathrm{Hg}$. All these changes, may lead to respiratory distress, compromised mental status, coma and death. DHF/DSS has a $5 \%$ mortality rate and children and elderly are at the greatest risk $^{5,6}$.

It is noteworthy that platelet deficiency is rarely the cause of death in people afflicted by this disease. The primary cause of death in patients suffering from dengue is the capillary leakage, leading to blood deficiency in the intravascular compartment, followed by multi-organ failure. Persistent vomiting decreased level of consciousness, severe abdominal pain and sudden decrease of body temperature to hypothermia are warning signs of serious condition of the patient.

Dengue is a self- limiting disease and recovery can take place between 2 to 7 days, however, there is no specific drug therapy. The main goal of treatment is to prevent shock, provide basic supportive measures which include giving fluids either by mouth or intravenously throughout the illness. Patients suffering from dengue should seek medical advice, rest and drink plenty of fluids in the form of juices and drinks. Paracetamol can be taken to bring down fever and reduce joint pains. NSAIDs should be avoided as they can affect the platelet function thereby leading to the risk of bleeding ${ }^{7}$. Platelet transfusion is usually not required unless the counts drop below 10,000 and there is some evidence of spontaneous and active bleeding At first instance of 
plasma leakage from the intravascular compartment to the extravascular compartment, fluid replacement amounting to $20 \mathrm{ml}$ per $\mathrm{kg}$ body weight per hour must be carried out and continued till the difference between the systolic and diastolic blood pressure is over $40 \mathrm{mmHg}$, or the patient passes adequate volume of urine. It has been suggested to utilize the formula of 20 , when treating patients with dengue. 'Formula of 20' i.e. rise in pulse by more than 20; fall of more than $20 \mathrm{mmHg}$ in upper and lower blood pressures and presence of more than 20 hemorrhagic spots on the arm after a tourniquet test, implies a high-risk situation and the patient would need immediate medical attention. Dengue can be diagnosed by detection of the virus in serum (serology) using ELISA, detection of IgM antibodies in blood by PCR (Polymerase Chain Reaction) etc. The best test for dengue is the DENGUE NS-1. The test is mostly positive from day 1 to day 7 after infection and cannot be false positive ${ }^{8}$.

In order to increase the number of platelets, certain herbal treatments have been documented. There is evidence that the aqueous extract of leaves of Carica papaya plant given 3 times a day would significantly increase the number of platelets ${ }^{9}$. However, in a recent systematic review and metaanalysis on the use of papaya leaves extract in dengue has questioned in terms of real efficacy of this treatment, and large scale clinical trials with focus on its role in preventing plasma leakage from intravascular compartment to extravascular compartment, prevention of shock and possible side-effects have been suggested ${ }^{10}$.

The only way to prevent dengue transmission is to combat the vector mosquitoes and limiting exposure to mosquito bites. It should be remembered that dengue breeds in clean stagnant water, therefore mosquito breeding places such as empty flower pots, open water tanks etc. should be either eliminated or properly covered. There should be provision of reliable water supply and regular garbage collection. Personal protection from mosquito bites can be achieved by using insecticide sprays in the house and mosquito repellents and covering most of the body parts especially after the monsoon season. Health education campaigns for the masses using the electronic and print media have always been helpful.
A novel vaccine for dengue has been prepared in a number of countries, however it is not commercially available as yet especially in the developing countries of South Asia ${ }^{11}$. Therefore, it is imperative that the health authorities in these countries must carry out emergency vector control programs and robust surveillance during disease outbreaks.

As per WHO, the recent ongoing outbreak of dengue in Pakistan has resulted in 47,120 confirmed cases and 75 related fatalities. In such a scenario, it is extremely important that the healthcare providers identify these cases early, provide necessary treatment and also educate the public at large on its prevention ${ }^{12}$.

\section{REFERENCES:}

1. Stanaway et al. The global burden of dengue: an analysis from the Global Burden of Disease Study 2013. Lancet Infect Dis 2016; 16(6): 712-23.

2. Brady et al. Refining the global spatial limits of dengue virus transmission by evidence-based consensus. PLoS Negl Trop Dis 2012; 6(8) e 1760 .

3. World health organization, emergencies, ten threats to global health in 2019. https://www.who.int/emergencies/ten-threatsto-global-health-in-2019.

4. Ikram Junaidi. Dengue outbreak sets new records in Pakistan. The DAWN, November 6, 2019.

5. Guzman MG, Harris E. Dengue. Lancet 2015; 385:453.

6. Deen JL, Harris E, Wills B, et al. The WHO dengue classification and case definitions: time for a reassessment. Lancet 2006; 368:170.

7. Rajapakse S, Rodrigo C, Rajapakse A. Treatment of dengue fever.Infect Drug Resist. 2012;5:103-112.

8. Halstead SB. Dengue. Lancet 2007; 370:1644.

9. Sarala N, Paknikar SS. Papaya extract to treat dengue: a novel therapeutic approach. Ann Med Health Sci Res 2014, 4(3); 320-324.

10. Rajapakse et al. Carica papaya extract in dengue: a systematic review and meta-analysis. BMC Complementary Medicine, October 11, 2019.

11. Rather IA, Parray HA, Lone JB, et al. Prevention and Control Strategies to Counter Dengue Virus Infection.Front Cell Infect Microbiol. 2017;7:336. Published 2017 Jul 25. doi:10.3389/ fcimb.2017.00336

12. World Health Organization, Disease outbreak news- Dengue fever - Pakistan. https://www.who.int/csr/don/19-november2019-dengue-pakistan/en/. 